\title{
EchoGéo
}

11 | 2010

Madagascar

\section{Des terres pour l'agro-industrie internationale ? Un dilemme pour la politique foncière malgache}

André Teyssier, Landry Ramarojohn et Rivo Andrianirina Ratsialonana

\section{OpenEdition}

Journals

Édition électronique

URL : https://journals.openedition.org/echogeo/11649

DOI : 10.4000/echogeo. 11649

ISSN : 1963-1197

Éditeur

Pôle de recherche pour l'organisation et la diffusion de l'information géographique (CNRS UMR 8586)

Référence électronique

André Teyssier, Landry Ramarojohn et Rivo Andrianirina Ratsialonana, « Des terres pour l'agroindustrie internationale? Un dilemme pour la politique foncière malgache », EchoGéo [En ligne], 11 | 2010, mis en ligne le 24 février 2010, consulté le 01 août 2021. URL : http://journals.openedition.org/ echogeo/11649; DOI : https://doi.org/10.4000/echogeo.11649

Ce document a été généré automatiquement le 1 août 2021.

EchoGéo est mis à disposition selon les termes de la licence Creative Commons Attribution - Pas d'Utilisation Commerciale - Pas de Modification 4.0 International (CC BY-NC-ND) 


\title{
Des terres pour l'agro-industrie internationale? Un dilemme pour la politique foncière malgache
}

\author{
André Teyssier, Landry Ramarojohn et Rivo Andrianirina Ratsialonana
}

1 Le 17 mars 2009, le président Ravalomanana cède le pouvoir dans des circonstances troubles, face à des contestations de rue violemment réprimées. La fermeture de la télévision du principal opposant 㡠le maire d'Antananarivo devenu ensuite président de la Haute Autorité de Transition歓, l'acquisition d'un nouvel avion présidentiel à l'origine en décembre 2008 de la suspension de l'aide budgétaire du FMI, le mécontentement d'opérateurs économiques offensés par les faveurs accordées au groupe agro-industriel Tiko et la dénonciation d'un projet de cession de 1.300 .000 ha de terres au groupe sud-coréen Daewoo auront eu raison d'un pouvoir légitimé par les urnes deux ans auparavant.

2 Se faisant l'écho à Madagascar d'un mouvement planétaire de manœuvres foncières à grande échelle ${ }^{1}$, "l'affaire Daewoo » a agité les médias du monde entier. Une tempête médiatique a soufflé sur la Grande-Ile, peu coutumière du fait et l'intérêt suscité par cette affaire a révélé d'autres projets similaires, notamment celui de la société Varun.

Ces projets d'appropriation ${ }^{2}$ à grande échelle ont heurté une opinion publique nationale et internationale émue par ce qui a été considéré comme des atteintes à la souveraineté nationale et au tanindrazana, la terre des ancêtres, et sont demeurés au stade de l'intention.

4 Les échecs de ces projets agro-industriels, vécus en même temps qu'une réforme foncière, peu médiatisée mais susceptible de favoriser un enregistrement massif des droits sur le sol, témoignent d'hésitations entre différents modèles de développement. Derrière l'emballement médiatique déclenché par ces projets, deux modèles de développement s'affrontent: l'un est basé sur le développement de l'exploitation familiale et des valeurs culturelles profondément ancrées, probablement ravivées pour l'occasion; l'autre est persuadé des vertus de «l'investissement direct étranger » et 
confiant dans l'effet d'entraînement économique supposé des filières agroindustrielles.

5 Pour contribuer au débat actuel, chargé d'idéologies, nous nous proposons de revisiter l'actualité de l'année 2009, afin de mieux comprendre le processus d'installation et le contenu de ces projets d'investissement. L'esprit initial de ces projets sera réexaminé et confronté aux principes fondateurs de la politique foncière malagasy, conduite par le même gouvernement depuis 2005. La mise en relief de ce paradoxe profond permettra de tirer des enseignements de nature stratégique pour l'accueil d'investissements sur la terre.

\section{Des tentatives illusoires d'appropriation foncière à grande échelle}

6 Les projets de Daewoo et de Varun ont "bénéficié » d'une couverture médiatique mondiale comme jamais l'agriculture malgache n'avait connu. Or, ces tentatives d'implantation agro-industrielle se sont réalisées en toute discrétion et le faible niveau d'informations ayant filtré invite à éclairer les processus qui ont conduit à leur suspension et, en partie, au renversement du régime.

7 Il apparaît d'ailleurs que ces montages sont différents, Daewoo ayant visé des appropriations à grande échelle tandis que Varun a privilégié des formes de contractualisation avec les occupants des terres convoitées.

\section{Daewoo : chroniques d'un projet fortement médiatisé et abandonné}

\section{Chronologie des faits}

8 Daewoo Logistics Corporation est un groupe sud-coréen spécialisé en logistique maritime, qui se diversifie à partir de 2006 dans l'agro-business (maïs, huile de palme, hévéa), notamment en Indonésie. Il apparaît à Madagascar comme l'un des prestataires en logistique du projet d'extraction de nickel d'Ambatovy, réalisé par un consortium associant le canadien Sherritt, le sud-coréen Kores et le japonais Sumitomo. Suite à cette première implantation, Daewoo Logistics ouvre en juillet 2008 une filiale de droit malgache à Antananarivo, Madagascar Future Entreprise (MFE), et dès septembre 2008, MFE lance un appel à recrutement pour plusieurs postes de directeurs régionaux. Fin octobre, une délégation des responsables des sociétés Daewoo, Kores et Posco est reçue par le président Ravalomanana et il est fait état de projets d'investissement dans les secteurs miniers et agricoles.

9 Le projet d'investissement foncier est rendu public le 18 novembre 2008, par un article du Financial Times ${ }^{3}$ qui relate une conférence de presse de Daewoo tenue la veille à Séoul. Le directeur financier du groupe y annonce que Madagascar cède gratuitement à sa société 1.300 .000 ha de terres durant 99 ans, pour produire $500.000 \mathrm{t}$ d'huile de palme dans les régions de l'Est et $4.000 .000 \mathrm{t}$ de maïs dans la partie occidentale de l'île, l'ensemble de la production étant destinée à l'exportation vers la Corée ou d'autres marchés. L'accès à ces terrains serait gratuit en contrepartie d'un investissement de 6 milliards \$ sur 25 ans $^{4}$. L'encadrement sera sud-africain et la main-d'œuvre non qualifiée recrutée localement. Le jour de cette annonce se tient au palais de Iavoloha le «Dialogue Présidentiel» sur le développement rural, sans aucune évocation de ce 
projet majeur. Plusieurs observateurs notent qu'il s'agit pourtant d'un projet d'appropriation foncière hors norme, sans doute l'un des plus importants jamais envisagés dans le monde.

10 A partir de cette date, Internet s'emballe, les agences de communication multiplient les dépêches, les médias du monde entier relaient ces informations ${ }^{5}$, tandis que les formules choc se succèdent en rafales ${ }^{6}$. La FAO évoque le retour d'un "système néocolonial $\aleph^{7}$, on compare la surface annoncée du projet avec celle de l'ile de France ou de la moitié de la Belgique. La spirale s'accélère et certaines informations se déconnectent progressivement des réalités. Ainsi, la moitié des terres arables malgaches serait concernée, on parle de louer la moitié de Madagascar,... Pour la presse mondiale, l'affaire est entendue et les terres sont déjà contrôlées par Daewoo8.

11 Ces informations sont ensuite reprises par des cercles militants de diverses obédiences, par des blogs de journalistes et par la diaspora malgache en Europe ${ }^{9}$, puis par des organisations rurales nationales, qui, à leur tour, renvoient des informations vers l'extérieur. Ce buzz accentue la pression à l'encontre d'un gouvernement embarrassé par les déclarations précipitées de Daewoo. Un démenti du Ministre de la Réforme Foncière, des Domaines et de l'Aménagement du Territoire daté du 25/11/08 est publié le lendemain dans la presse nationale. Ce communiqué informe d'une "convention de prospection préliminaire» et nie formellement l'attribution de terres à Daewoo ${ }^{10}$. Il rappelle que des demandes d'acquisition de telles surfaces doivent faire l'objet d'une approbation en Conseil du Gouvernement.

Il s'avère néanmoins que des procédures « d'acquisition de terrains domaniaux » ont été engagées pour des portions de plusieurs centaines de milliers d'hectares : 170.000 ha demandés auprès des services des Domaines de Toamasina, 336.000 ha dans la région du Melaky ${ }^{11}, \ldots$ Des variétés de maïs ont fait l'objet d'expérimentations dans le Menabe et des contrats de production ont été préparés. Au-delà du simple accord de prospection, il apparait que les bases d'un projet aient été effectivement mises en œuvre. Ce projet devait d'ailleurs entrer dans un cadre de coopération plus large entre la Corée du Sud et Madagascar, combinant aide publique et investissements du secteur privé $^{12}$.

\section{Les « Pour » et les « Contre »}

Dès les intentions de Daewoo portées à la connaissance de l'opinion publique, de vives réactions, diamétralement opposées, se sont fait entendre.

Les partisans de la cession de terres aux capitaux étrangers comptent les tenants d'un discours libéral qui s'appuie sur des déclarations d'experts, les responsables des entreprises sud-coréennes concernées et un certain regard tananarivien sur le monde rural malgache. Selon eux, le développement passe par le seul investissement privé qu'il faut activement encourager ${ }^{13}$, notamment dans l'agriculture car les sols malgaches seraient largement sous-exploités ou mis en valeur de façon rudimentaire par des paysans dont on déplore l'archaïsme des pratiques culturales. On retrouve ici les sempiternels jugements, hérités de l'époque coloniale mais toujours audibles dans la capitale, sur "l'élevage contemplatif», la dévastation des forêts et le recours systématique au feu par une paysannerie prédatrice et inconsciente, et sur ces exploitations agricoles qui stagnent dans un système d'autosubsistance. 

pour le moins approximatives. En 2007, les superficies cultivées occuperaient, selon la FAO, 3,55 millions d'hectares sur 15 à 20 millions de terres arables ${ }^{14}$, tandis que d'autres experts confirment avec une précision déconcertante que « $3,53 \%$ des terres cultivables sont cultivées $»^{15}$. Ce pourcentage, variable de 3 à $8 \%$ selon les sources, interpelle quiconque ayant connaissance des terroirs malgaches sur la localisation des $97 \%$ restants de terres cultivables mais non cultivées... L'observation des paysages ruraux des Hautes Terres et une imposante littérature scientifique ${ }^{16}$ montrent a contrario la capacité des sociétés paysannes malgaches à aménager des terrains apparemment non cultivables en terres cultivées. L'occupation plus lâche des sols des régions côtières ou intermédiaires, moins densément peuplées, peut paraître propice à l'installation de grands projets agro-industriels, mais les sols fertiles et arrosés des régions de l'Ouest malgache ne se trouvent que dans de grandes vallées alluviales déjà occupées, sinon immatriculées depuis la période coloniale. La façade orientale présente également des densités de population importante et un relief tourmenté, peu favorable à une grande agriculture mécanisée.

16 Ces chiffres traduisent un certain flou sur les concepts et une caractérisation insuffisante de l'occupation réelle des terres à Madagascar. Ils entretiennent le mythe de terres vierges et immenses, vides de droits et disponibles pour toute transaction. Les dirigeants de Daewoo et la presse sud-coréenne adhèrent à la vision qui leur est servie et perçoivent les informations des médias européens et américains comme autant d'agressions : nous contribuons à développer Madagascar en transformant des «terres en friche» en espaces de production agro-industrielle grâce à des investissements massifs et nous sommes critiqués par d'anciennes puissances coloniales en perte d'influence ${ }^{17}$. Ces arguments sont relayés plus récemment par quelques chroniqueurs de quotidiens nationaux qui remettent en cause le bien-fondé de valeurs telles la souveraineté nationale ou la terre des ancêtres quand une majeure partie de la population vit au-dessous du seuil de pauvreté ${ }^{18}$.

Les opposants au projet Daewoo les plus offensifs sont constitués de diverses personnalités ${ }^{19}$, d'ONG et d'organisations de la diaspora, notamment le Collectif pour la Défense des Terres Malgaches, disposant de forums internet où sont échangés des propos extrêmement virulents ${ }^{20}$. Les organisations rurales nationales sont moins bruyantes, mais il faut néanmoins signaler la remise au gouvernement d'un communiqué conjoint ${ }^{21}$ faisant état d'inquiétudes sur le devenir de l'agriculture paysanne et «du contrôle de surfaces agricoles importantes par une entreprise étrangère ». Ces préoccupations sont reprises par quelques groupes de réflexion dont le Sefafi qui publie le 08/12/08 un communiqué retentissant, dénonçant « un nouvel avatar colonial dépouillant la Grande-Ile de l'une de ses principales richesses naturelles, avec la complicité de ses gouvernants $\gg .^{22}$

18 Les arguments des opposants au projet Daewoo rappellent l'enjeu stratégique de la souveraineté alimentaire (peut-on exporter massivement des denrées agricoles quand le PAM apporte une assistance alimentaire à un demi-million de personnes chaque année $^{23}$ ?) et s'interrogent sur la portée réelle des retombées économiques. Les 70.000 recrutements annoncés par Daewoo ne représenteraient qu'un emploi sur 18,5 ha quand 0,8 ha permet actuellement la survie d'une famille. Mais surtout, ces propos attisent un nationalisme latent: l'Etat brade le patrimoine et brise un tabou (d'Esru, 2009) en cédant le tanindrazana aux étrangers ${ }^{24}$, il accorde des traitements de faveur aux 
étrangers quand les ressortissants malgaches éprouvent les pires difficultés pour faire reconnaître leurs droits sur la terre. Ces arguments frappent la sensibilité nationale et vont alimenter la montée en puissance de la contestation contre le pouvoir en place.

\section{Daewoo utilisé comme levier de déstabilisation}

19 "L'affaire Daewoo » devient ainsi le « détonateur de l'insurrection » ${ }^{25}$. Elle va permettre un élargissement et une intensification de la mobilisation contre le régime Ravalomanana sur le thème infaillible de la «trahison à la patrie $»^{26}$. A partir de décembre 2008, au cours des manifestations qui se multiplient dans le pays comme à l'extérieur, sont scandés des slogans hostiles au projet Daewoo. A cette date, la société coréenne commence à douter ${ }^{27}$ et à la mi-janvier, le projet semble déjà enterrée ${ }^{28}$. D'ailleurs, des topographes évitaient déjà de réaliser les travaux de terrain pour le compte de Daewoo, en prétextant diverses pannes de matériel. L'opposition n'en reste pas là et demande le 16 janvier la démission du ministre de la Réforme Foncière et des Domaines ${ }^{29}$. Fin janvier 2009 à Toamasina, des manifestations se sont dirigées vers le service des Domaines et certains agents, accusés par la foule de vendre la terre aux étrangers, se sont prudemment repliés sur la capitale.

Le 18 mars 2009, au lendemain de sa prise de pouvoir, le tout nouveau président de la Haute Autorité de Transition, M. Andry Rajoelina, évoque la Constitution pour justifier que les terres à Madagascar ne seraient «ni à vendre ni à louer» et confirme la suspension du dossier Daewoo. Quatre mois plus tard, Daewoo Logistics fait faillite, en raison de la diminution du trafic maritime mondial, de graves problèmes de trésorerie et... de l'échec de l'implantation à Madagascar ${ }^{30}$. Le nouveau ministre en charge du secteur foncier confirme l'arrêt définitif du projet Daewoo le 10 avril tout en précisant que Madagascar reste ouvert à tout projet d'agro-business ${ }^{31}$. A cette époque, le cas de la société Varun n'est pas encore statué.

\section{Varun : un projet d'agriculture contractuelle?}

21 C'est également un article de la presse internationale ${ }^{32}$ qui dévoile quatre jours après le renversement $\mathrm{du}$ pouvoir, un projet portant sur 465.000 hectares initié, plus discrètement, par Varun, une société indienne dont le siège est implanté à Bombay. Sa filiale malgache, Varun International Madagascar SARL, est une entreprise d'importexport de marchandises générales et de produits miniers ${ }^{33}$. Elle ne présente pas de compétence particulière en aménagement rural ou en lotissement urbain.

\section{Un projet mixte d'appropriation de terres domaniales et de contrats de production}

Suite à une rencontre en janvier 2008 entre le président de Varun Industries et le président Ravalomanana, et la signature d'un mémorandum avec le Ministère de l'agriculture en septembre 2008, un protocole d'accord est signé le 25 octobre 2008 entre la région Sofia et Varun. Le projet de Varun est présenté comme un projet de production agricole, de réalisations hydro-électriques et de création de villes nouvelles, avec une perspective de création d'emplois et de contribution à l'indépendance alimentaire. La région s'engage à "mettre à disposition $d u$ "partenaire " les terrains disponibles et aménageables pour une durée de 50 ans » et sur une surface de 130.000 ha. Une convention de partenariat précise l'accord en décembre 2008 : dans « 13 plaines » 
repérées, la fourniture de terrains par la région se limite à un appui aux négociations avec les agriculteurs et à une assistance aux procédures d'acquisition de terrains domaniaux, tandis que l'engagement de Varun à créer des villes nouvelles et à construire des infrastructures hydro-électriques se transforment respectivement en "lotissement low cost» et en prises de contact avec des entreprises spécialisées. L'instruction de ce projet se solde le 26 janvier 2009 par un atelier de signature à Antsohihy du "contract farming", auquel assistent 250 personnes, représentant les autorités régionales et des producteurs des 13 plaines.

Ce projet reprend le vocabulaire habituel des projets d'aide au développement : on parle de "Grands Périmètres Irrigués» et de "bénéficiaires de projet». Il s'étend initialement sur une superficie initiale de 130.000 ha, mais les premiers repérages de terrain étendent les surfaces à 232.000 ha. Varun reconnaît que 3/4 des terres convoitées sont occupées. Aussi, le projet comprend l'obtention de 61.000 ha de terrains domaniaux en bail emphytéotique et des contrats de production avec les agriculteurs sur les 171.000 ha déjà occupés.

Les négociations pour l'accès aux terrains sont confiées à un bureau d'études national, Sodhai. La mission confiée par Varun à ce bureau d'études relève de l'impossible: il s'agit de créer 13 groupements paysans (un par plaine, dans six districts) et de négocier des contrats de location, d'engager les procédures d'acquisition de terrains domaniaux et de baux emphytéotiques, de réaliser les travaux topographiques en conséquence et d'organiser un « atelier de signature " pour validation du projet par ces tout nouveaux groupements, le tout en deux semaines et en une seule mission de terrain ${ }^{34}$.

Le projet de Varun est particulièrement ambitieux. L'investissement total s'élève à 1,170 milliard \$ pour aménager 13 périmètres irrigués en 2 ans. L'objectif est de parvenir à produire 2,800 millions tonnes de paddy ${ }^{35}$ et 400.000 tonnes de maïs par an dès la quatrième année. $20 \%$ du riz, $50 \%$ du maïs, $100 \%$ du dal ${ }^{36}$ produits seront exportés. Il est espéré une fabuleuse augmentation des rendements de paddy, de 3 à 10 ou 12 tonnes par hectare, et de $4 \mathrm{t}$ pour le maïs, grâce à la mécanisation et à une intensification systématique. Le retour sur investissement est escompté dès la troisième année...

Les contreparties apparaissent moins clairement dans les documents de projet de Varun. Si les infrastructures du "projet» (bureaux, logements du personnel, ateliers, garages,...) sont listées en détail, en revanche, les cases de santé, les écoles, les réseaux d'électricité et d'eau potable, sont annoncés sans être quantifiés. Sur les terres occupées, les termes de ces « contract farming " révèlent des accords dont la mise en œuvre aurait été délicate.

\section{"Contract farming " : une forme déguisée d'accaparement}

Ces contrats ne sont pas passés avec chacun des agriculteurs ayant-droits. Les accords sont signés entre Varun et les 13 " plaines » constituées en associations pour l'occasion par Sodhai, représentées par leurs présidents. Ces responsables sont supposés être habilités à «donner de la terre à Varun pour des cultures » sur 171.000 ha Ils s'engagent non seulement en leur nom et pour leurs propres terrains, mais également au nom et pour les terrains de leurs descendants et des "propriétaires de plein droit » membres de l'association, cela pour des locations de terres d'une durée de 50 ans ! ${ }^{37}$ 
Ces contrats prévoient que les 13 associations autorisent Varun à occuper les terres et à réaliser tous les travaux agricoles, que les terres concédées ne feront l'objet d'aucune revendication et d'aucun conflit, et que Varun puisse vendre la production en toute liberté. Ils soumettent les détenteurs de terrain à une clause de confidentialité et les engagent à ne pas interférer dans les travaux de Varun. Varun se charge de la fourniture d'équipements et d'intrants, de l'installation d'infrastructures sociales ${ }^{38}$ et du recrutement d'une main-d'œuvre de préférence locale. Varun n'a pas de contact direct avec les associations paysannes: le bureau d'études Sodhai représente les associations pour toute transaction avec Varun.

La répartition de la production envisagée par ces contrats en montre le caractère inique. $70 \%$ de la production revient à Varun. Sur les $30 \%$ de récoltes revenant au détenteur des terrains, $70 \%$ doivent obligatoirement être vendus à Varun, au cours fixé par l'entreprise.

30 La portée de ces contrats reste floue. Varun, peu satisfait des délais de contractualisation, déplore les lenteurs procédurières de l'administration foncière. La société espérait pouvoir considérer «l'atelier de signature » comme la validation des contrats. La tournure des évènements s'y opposera. Le même jour, les émeutes à Antananarivo marquent le début des manifestations et le projet est resté en suspens depuis cette date ${ }^{39}$. Plusieurs déclarations gouvernementales ont confirmé la décision de suspendre tout projet d'acquisition foncière et de réduire à 20 ans la durée des baux emphytéotiques, mais aucune disposition légale n'est venue formaliser ces intentions ${ }^{40}$.

\section{Une contribution au développement contestable}

31 La "hausse de revenus des paysans " annoncée par Varun est pour le moins discutable. Même si Varun reconnaît les droits fonciers des occupants et se défend de toute entreprise de spoliation ${ }^{41}$, les contrats de fermage envisagés risquaient de provoquer davantage de pauvreté et d'exclusion, tandis que les retombées attendues semblaient limitées à des effets d'annonce.

Même avec un rendement illusoire de 10 tonnes de paddy par hectare et selon les termes des contrats, une famille paysanne disposant de 1 ha, et qui compte en moyenne 5 personnes, aurait perçu une rente annuelle de 3 tonnes de paddy dont $900 \mathrm{~kg}$ en nature, soit $585 \mathrm{~kg}$ de riz blanc. Or, une famille rurale consomme environ $700 \mathrm{~kg}$ de riz blanc par an. Elle aurait dû nécessairement acheter du riz, sur un marché contrôlé par Varun.

33 Il se pose également une question d'activité pour ces paysans convertis de gré ou de force en rentiers. Les ouvriers de Varun réalisant les travaux agricoles, quelle activité restera-t-il à ces familles dorénavant sans terre ? Peuvent-elles conserver une activité agricole si les terres sont à la disposition de Varun pendant un demi siècle ? Doiventelles se préparer à la migration? Vers de nouvelles terres à conquérir, probablement en défrichant les dernières réserves foncières que sont les reliques forestières? $\mathrm{Ou}$ doivent-elles se déplacer, sans aucune qualification, vers des villes dont les maigres secteurs de l'industrie et des services sont peu généreux en emplois?

34 Dans la précipitation du montage de ce projet, ces questions fondamentales n'ont pas été abordées. Les Plans de Développement des communes concernées et les conclusions des différentes concertations sur le développement de la région Sofia ont été ignorées. D'ailleurs, plusieurs élus et responsables régionaux se sont interrogés dès le début de ce 
projet sur la réalité de ses retombées économiques et sociales. La construction de barrages et de canaux d'irrigation, de routes, d'hôpitaux et d'écoles, a été annoncée ${ }^{42}$ sans qu'il ne soit possible d'en trouver la moindre trace dans les documents du projet ${ }^{43}$.

$\mathrm{Au}$ fur et à mesure de la préparation du projet, l'enthousiasme des premières rencontres avec les autorités malgaches revient vers des objectifs plus modestes. Ainsi, on passe de villes nouvelles à des programmes de logement social et il n'est fait état ni de barrages, ni d'aménagement hydro-agricoles dans les accords avec les associations paysannes. L'ampleur des recrutements reste faible, ce qui est cohérent avec la logique d'un projet de grande culture mécanisée. L'embauche prévue de 1.500 personnes correspond à un emploi pour 155 ha ; une telle perspective s'inscrit largement en-deçà des capacités de charge de l'agriculture familiale.

\section{Des projets d'emblée compromis}

Les déboires de Daewoo et de Varun montrent que des projets d'une telle envergure sont irrémédiablement condamnés à l'échec ${ }^{44}$. Non seulement leur faisabilité technique et financière s'avère des plus incertaines, mais la dimension juridique de leur mise en œuvre se heurte aux principes fondateurs d'une nouvelle politique foncière à tel point que l'instruction de ces projets, malgré l'ampleur des changements qu'ils introduisent, a été conduite, tant que faire se peut, dans la plus grande discrétion.

\section{Une opacité rendue nécessaire par la finalité des projets}

De toute évidence, les promoteurs de ces deux grands projets d'appropriation foncière ont douté de leur acceptation sociale. Les contrats de Varun, par leur recherche de confidentialité et d'engagements à respecter l'entreprise, son personnel et son matériel, révèlent une crainte de conflits sociaux et espèrent contenir suffisamment de clauses pour protéger les contrats agraires de probables contestations.

Aussi, ces projets sont instruits "en cachette ». Très peu d'informations sont communiquées, que ce soit auprès des populations concernées ou auprès du grand public. Aucune volonté de concertation et d'explicitation des enjeux et du contenu des projets ne transparaît réellement. Au contraire, les autorités locales, et notamment les chefs de district, sont tenus de " faire accepter le projet $»^{45}$. Cette opacité dans le montage des projets a accrédité l'idée que "le chef de l'Etat dirige le pays comme il gère son entreprise $»^{46}$ et a laissé toute latitude aux rumeurs et aux suspicions. On pourrait évoquer un comportement social propre à Madagascar où le non dit serait élevé au rang de vertu. Il apparaît surtout que les conséquences de ces projets étaient indicibles.

Si l'implantation de Daewoo dans les districts enclavés et faiblement peuplés de la région Melaky pouvait se concevoir sans procéder à des déguerpissements massifs, il semble en revanche qu'une culture industrielle du palmier à huile dans les régions de la SAVA et d'Atsinanana ne puisse se développer sur 300.000 ha sans se déployer sur les emprises cultivées de dizaines de milliers d'exploitations familiales. La prise de contrôle de terres occupées paraissait inévitable. Comment alors rendre transparents les objectifs d'un projet par essence inacceptable?

Les promesses de compensation n'ont pas suffi, tant la crainte de dépossession des terres est forte. Varun est allé jusqu'à proposer un appui à l'obtention de titres de 
propriété, voire la création de guichets fonciers communaux, au profit des détenteurs de parcelles qui accepteraient les contrats de fermage. Ces perspectives n'ont pas rassuré une population attachée à ses terres et qui se voyait priver de leur usage pendant 50 ans.

\section{Faisabilité technique et concurrences sur l'espace}

41 Il a sans doute été vendu à Daewoo et à Varun le mythe de "terres vacantes et sans maître ». Ces deux sociétés se sont rapidement trouvées confrontées à des réalités de terrain bien différentes.

Les espaces les plus propices à une activité agro-industrielle sont déjà occupés et mis en valeur, soit par des exploitations paysannes, soit par des entreprises agricoles déjà installées. Les espaces disponibles correspondent à des terres dont les caractéristiques physiques présentent des contraintes agronomiques, en eau et en fertilité, ou à des zones enclavées dont l'accès nécessiterait au préalable des chantiers conséquents de constructions de routes et de ponts.

43 Les faibles densités démographiques des régions côtières de l'Ouest malgache peuvent donner l'impression que des espaces restent disponibles pour des implantations agroindustrielles. Il convient de nuancer cette illusion statistique. En réalité, la répartition de la population se concentre sur les espaces « utiles » que sont les grandes plaines alluviales du Mangoky, de la Morondava, de la Manambola ou de la Tsiribihina,... Ces plaines, déjà aménagées et appropriées, laissent peu de place à de nouveaux acteurs, surtout s'ils apparaissent comme de gros consommateurs de terres.

Quel que soit le niveau d'occupation des terres, des droits sur le sol, formalisés par écrit ou non, couvrent de manière quasi-systématique l'espace rural malgache. Les grandes plaines alluviales des régions côtières sont en grande partie immatriculées au nom d'anciennes agro-industries établies à l'époque coloniale, les collines de l'Est sont appropriées par des lignages familiaux depuis plusieurs générations quand les savanes de l'Ouest sont réparties en droits pastoraux.

Restreintes par les droits existants, les ambitions des projets d'appropriation foncière à grande échelle se voient également contingentées par une politique de démultiplication des aires protégées ${ }^{47}$ et par les stratégies de colonisation de migrants venant des Hautes Terres ou des régions du Sud de l'île. Elles peuvent être également gênées par les servitudes à libérer pour les projets d'extraction minière et de prospection pétrolière.

46 A défaut d'une politique d'aménagement du territoire conçue en concertation avec les acteurs régionaux et locaux ${ }^{48}$, une compétition croissante va s'exercer pour le contrôle de l'espace entre différents secteurs d'activités et entre groupes d'acteurs. Les tentatives de dynamisation de l'économie par l'attraction de capitaux internationaux, tout comme les tentatives de préservation de la biodiversité, se heurteront inévitablement à la légitimité des occupations des populations résidentes, tant que celles-ci seront écartées des choix de développement et qu'elles ne ressentiront pas concrètement les bénéfices de ces choix.

\section{Une faisabilité juridique contrariée par la réforme foncière}

Pour la conduite de leurs projets, Daewoo et Varun ont eu pour interlocuteurs les Régions concernées, l'Economic Development Board of Madagascar (EDBM) ${ }^{49}$, le 
Ministère de la Réforme Foncière, des Domaines et de l'Aménagement du Territoire et ses services déconcentrés. Les démarches initiées par les deux sociétés se sont engagées dans le respect de la législation malgache, et notamment de la loi sur les

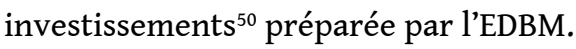

Cette loi adoptée en décembre 2007 fournit le cadre légal à une politique de développement basée sur l'ouverture aux capitaux étrangers. Ce texte aux allures de manifeste libéral entend «rendre très attractif le climat des affaires " par des avantages fiscaux et par l'application de principes indiscutables, tels la liberté d'investissement, l'égalité de traitement entre les investisseurs, la protection des droits de propriété contre toute mesure de nationalisation ou d'expropriation, ou la liberté de transferts de fonds. Cette loi sur les investissements élargit les possibilités d'accès à la terre pour les personnes physiques et morales étrangères, soit par acquisition, sous réserve d'obtenir de l'EDBM une "autorisation d'acquisition foncière", ce qui modifie radicalement les dispositions précédentes ${ }^{51}$. L'EDBM est ainsi devenue, pour le compte de la Présidence, l'institution chargée de faciliter l'accès au sol pour les investisseurs, ce qui atteste d'une volonté de gérer les grands investissements sur la terre par le plus haut niveau de l'Etat.

Néanmoins, la valeur juridique de ces autorisations reste ambiguë : elles ne constituent pas un titre foncier, elles ne consacrent pas de droit de propriété, mais elles peuvent faire l'objet d'une cession. Elles permettent à des étrangers, selon une procédure simple et rapide ${ }^{52}$, d'engager une procédure d'immatriculation foncière selon les procédures habituelles de la Direction des Domaines. Sans doute ces autorisations auraient été considérées comme des "feux verts» pour engager immédiatement une activité productive ou commerciale sans avoir à attendre le délai nécessaire à une immatriculation. Les investisseurs auraient ainsi bénéficié d'un traitement accéléré grâce à une procédure qui se serait contentée d'une autorisation de l'EDBM en attendant la formalisation de la propriété par immatriculation. Mais le décret d'application de cette loi n'est pas paru et il semble qu'aucune autorisation n'ait été délivrée à ce jour. Daewoo et Varun ont donc été orientés vers des baux emphytéotiques.

50 La phase initiale de ces grands projets a donc été facilitée d'un point de vue administratif par ce dispositif légal mais les principales contraintes n'ont pas été levées: les questions opérationnelles et celle de l'appartenance des terrains non titrés restent entières.

51 Les baux emphytéotiques sont des contrats de location. Leur établissement demande à ce que le titulaire de la terre soit clairement identifié par immatriculation et bornages préalables. Les terrains repérés par Daewoo et Varun ne sont pas d'un seul tenant et s'étendent sur des superficies immenses. Leur délimitation exige des travaux topographiques de grande ampleur, ce dont l'administration foncière est aujourd'hui dans l'incapacité de réaliser dans des délais acceptables pour ces entreprises, tant ses ressources et sa capacité d'intervention ont été réduites ${ }^{53}$. Rappelons qu'il a fallu 60 ans à la colonisation française pour implanter 1.091 .000 ha de concessions (Raison, 1969).

Le déploiement de ces baux emphytéotiques sur de grandes surfaces se serait immanquablement étendu sur des terrains appropriés selon des usages ancestraux. L'idée de titrer ces terrains au nom de l'Etat pour ensuite contracter un bail emphytéotique avec Daewoo relève d'une contradiction majeure avec la nouvelle politique foncière mise en place par le même gouvernement. Depuis octobre 2005, les 
terres non immatriculées mais occupées ne relèvent plus du domaine privé de l'Etat; elles sont désormais présumées comme des propriétés privées à part entière. L'Etat n'a plus la possibilité d'en disposer et encore moins de les attribuer. A moins d'ignorer ces dispositions récentes, la faisabilité juridique des projets fonciers de Daewoo et de Varun s'avérait très complexe, sinon compromise.

\section{Les paradoxes d'une politique foncière comme reflets de modèles de développement hésitants}

53 L'appui de l'Etat à l'installation de Daewoo et de Varun a montré la persistance de réflexes domaniaux au sein des pouvoirs publics, comme peuvent en attester les engagements de plusieurs régions à "fournir des terrains" à ces entreprises. De telles décisions ne sont plus conformes au cadre légal en vigueur car la présomption de domanialité est supprimée depuis octobre 2005.

\section{Une réforme basée sur la décentralisation de la gestion foncière}

Le système foncier malgache calqué il y a 110 ans sur le Torrens Act australien (Teyssier et al., 2008) repose sur un principe fondamental : toutes les terres sont supposées appartenir à l'Etat tant que les occupations de terrain n'ont pas fait l'objet d'une immatriculation au registre foncier. La propriété privée ne peut être consacrée que par la délivrance d'un titre foncier à l'usager qui aura suivi cette procédure. Depuis plus d'un siècle d'administration des terres par l'Etat, moins de 500.000 parcelles ont été immatriculées et autant restent en instance, parfois depuis plusieurs décennies. Pour obtenir ce titre, l'usager doit payer l'équivalent de 500 \$ et s'engager dans une procédure qui dure en moyenne 6 ans (ECR, 2006, 2008). Ces coûts et délais éloignent une grande majorité d'usagers, notamment en milieu rural, d'une possible reconnaissance de leurs droits sur la terre. Compte tenu d'un besoin grandissant d'écrire les droits, un système local d'enregistrement des transactions, fait de différents "petits papiers", s'est développé en conséquence dans l'ensemble du pays, indépendamment de la documentation foncière gérée par les services domaniaux. Ainsi, les titres conservés par l'administration sont rarement actualisés et ne correspondent pas souvent aux occupations actuelles.

Face à l'inertie de ce système, le Gouvernement malagasy s'est engagé dans une réforme avec l'objectif de répondre «à la demande massive en sécurisation foncière, dans de brefs délais et à des coûts ajustés au contexte économique, par la formalisation des droits fonciers non écrits ${ }^{54}$. La solution proposée, qui s'inspire largement de la pratique sociale, est radicale. Pour enregistrer et garantir les droits fonciers, une série de nouvelles lois et décrets ${ }^{55}$ attribue aux communes une nouvelle compétence: elles sont désormais habilitées à reconnaître et à gérer la propriété privée non titrée, c'est-àdire l'ensemble des terrains objet d'une "emprise personnelle", mais encore non immatriculés. La commune peut s'équiper d'un "guichet foncier communal ", apte à délivrer des " certificats fonciers " d'une valeur juridique quasiment identique à celle du titre foncier ${ }^{56}$ (Teyssier et al., 2007, Rochegude 2008). La procédure de certification est publique et contradictoire. A la demande des usagers, des "commissions de reconnaissance locale » composées de représentants de la commune, du fokontany et d'au moins deux ray-amand'reny ${ }^{57}$, procèdent à la reconnaissance des terrains en 
présence du voisinage, tentent de régler les éventuelles oppositions et établissent un procès-verbal à destination du maire, chargé de la validation finale du certificat. Ce pari sur la compétence locale prend pour hypothèse que la gestion foncière gagnera en efficacité et en équité si elle est rapprochée des usagers et placée sous la responsabilité des collectivités locales (Teyssier et al., 2009).

L'engouement des communes malgaches pour cette nouvelle orientation de politique foncière, renforcé par l'appui de la communauté internationale ${ }^{58}$, a provoqué un développement rapide des guichets fonciers dans un délai relativement court. Ainsi, de 2006 à 2009, 300 communes se sont équipées d'un guichet foncier et 150 sont en cours de montage ${ }^{59}$. Ces communes ont enregistré sur la même période 107.000 demandes de certificats et en ont délivré 45.000 , un chiffre à rapprocher des 400 à 500.000 titres fonciers établis dans l'ensemble du pays depuis plus d'un siècle. Le démarrage de la réforme foncière est ainsi devenu l'un des points forts de l'action gouvernementale.

Ce nouveau pouvoir de gestion foncière transféré à l'échelon local et la suppression du principe de domanialité réduisent aujourd'hui la latitude de l'Etat à dégager des terres pour les investisseurs sans avoir à considérer les populations en place. L'Etat dispose d'un domaine public ${ }^{60}$ et d'un domaine privé réduit désormais aux terrains immatriculés au nom d'un acteur public et aux terrains effectivement libres de toute occupation $^{61}$. Il ne peut plus, théoriquement, intervenir à sa guise sur des terrains relevant de la propriété privée non titrée. Cette réorientation de politique foncière, censée protéger les droits des occupants actuels, devrait obliger les grands projets agro-industriels à composer avec les détenteurs de droits préexistants. Elle devrait aboutir à une modification du « comportement domanial » des autorités centrales. Or, ce système domanial convenait davantage aux visions ébauchées récemment pour le développement de Madagascar.

\section{L'espoir d'un bond en avant libéral et futuriste}

58 Les projets de Daewoo et de Varun ne sont que les parties immergées d'un modèle de développement particulièrement ambitieux. Ils s'inscrivent dans une vision plus large, inspirée de travaux d'économistes ${ }^{62}$, qui privilégiait la création de "cités portail $»^{63}$ dans une aire extraterritoriale, administrées par une autorité internationale et supposées concentrer l'investissement étranger grâce à des régimes fiscaux et législatifs peu contraignants (Romer, 2008). A l'image de Hong-Kong, présenté (avec Dubaï...) comme un modèle, le développement d'une ville nouvelle au sein d'une zone internationale créée ex-nihilo ${ }^{64}$ va entraîner une dynamique économique. Des projets d'agro-business seront initiés dans l'hinterland de ce pôle qui consommera et exportera la production agricole.

59 La proposition de coopération sud-coréenne de décembre $2008^{65}$ s'inscrit dans cette perspective. Un modèle de développement sud-coréen est vendu : il s'appuie sur des projets futuristes de villes nouvelles "clé en main" dans le cadre de programmes d'investissement lourds, intégrant des politiques d'aménagement du territoire et d'enregistrement des terres par des dispositifs cadastraux de haute technologie.

L'émergence de cette vision futuriste apparaît à partir de 2008 et va bien au-delà des orientations du Madagascar Action Plan ${ }^{66}$. Ce plan quinquennal, qui a succédé à partir de 2007 au classique Document Stratégique de Réduction de la Pauvreté, retenait comme modèle de développement rural les principes de la "révolution verte", 
habituellement basés sur l'augmentation de la productivité et sur une production agricole orientée vers le marché. Ce modèle aspire à une transformation des exploitations familiales en entreprises rurales et à une évolution «de l'économie de subsistance vers l'économie de marché ${ }^{67}$. Il paraissait déjà en discordance avec les stratégies d'exploitations familiales, qui, sans être rétives à l'innovation, doivent gérer une multitude de risques dans des environnements physique et institutionnel incertains (Rakoto Ramiarantsoa, 2008).

61 Le modèle préconisé depuis se présente plutôt comme une projection utopique dans un avenir lointain et s'annonce encore plus distant des réalités paysannes. Les espoirs de «villes portail», de campagnes remodelées par de grands investissements agroindustriels, de petits cultivateurs transformés en ruraux vivant de rentes ou en nouveaux urbains, semblent faire fi d'un milieu humain peu disposé à ces évolutions. Ils s'opposent à un modèle de développement basé sur la promotion de la paysannerie et le respect de ses droits, notamment sur la terre.

62 La politique foncière révèle la confrontation d'un modèle de développement fascinant, rêvé en haut lieu et présenté à mots couverts, face à l'attente de solutions pragmatiques d'une société à dominante rurale et pauvre. Ainsi, on cherche à rassurer l'exploitation familiale par le certificat foncier pour préserver les droits sur la terre, tout en décidant, plus ou moins discrètement, de dispositions urgentes pour libérer des terrains susceptibles d'attirer du capital et de développer des filières agro-industrielles d'exportation. Ainsi, la mise en œuvre de cette politique reflète la permanence d'une vision tiraillée par une ambiguïté de fond et source d'incohérences : la décentralisation de la gestion des terres non titrées s'installe progressivement tandis que la délivrance d'autorisations d'occupation foncière pour les investisseurs est centralisée au plus haut niveau de l'Etat.

\section{Dépasser la dualité tanindrazana contre investissement?}

63 La protection des droits fonciers existants et l'ouverture des terres aux capitaux étrangers sont-elles pour autant deux stratégies politiques et économiques incompatibles? Le développement est-il conditionné à la seule cession de terres à des capitaux internationaux ou à la seule sécurisation des droits existants? Le respect du tanindrazana oblige-t-il à un usage des terres exclusivement réservé aux natifs ?

Cette dualité s'avère bien trop réductrice. Si l'on craint un risque évident d'exclusion des populations rurales confrontées à des projets agro-industriels démesurés comme ceux de Daewoo et de Varun, on peut également douter des performances d'une agriculture familiale, qui, jusqu'à présent et dans ses formes actuelles, maintient des centaines de milliers de familles dans une grande précarité. Il y a fort à parier que l'agriculteur pourrait se satisfaire d'entreprises d'agro-business de dimension raisonnable qui créeraient effectivement des emplois ou inciteraient à des productions de rente sans perdre de terres et selon des contrats équitables. Chacun des modèles présente sa part de faiblesses et d'incertitudes ; aucun ne devrait être défendu en l'état.

Des combinaisons entre les modèles sont-elles concevables ? Peut-on concevoir l'ouverture du monde rural malgache à la mondialisation tout en protégeant les droits fondamentaux d'une petite paysannerie sur ses terres ? Quel mode de régulation publique pourrait équilibrer des négociations particulièrement asymétriques pour éviter des processus d'appauvrissement et de ségrégation? 
66 matériau de la rhétorique politique. Certaines organisations militantes rejettent en bloc l'idée de formules "gagnant-gagnant» et bien des chercheurs s'interrogent sur les vertus effectives de combinaisons entre promotion de l'agriculture familiale et grands projets d'appropriation foncière ${ }^{68}$. Les connaissances sur l'intégration des agroindustries dans le tissu économique des campagnes malgaches ne suffisent probablement pas à éclairer un débat empreint d'idéologie. Si elle était légitimement investie pour aborder cette question, la recherche pourrait apporter, par la caractérisation des processus d'appropriation foncière et par l'évaluation des impacts environnementaux, sociaux et économiques, les éléments nécessaires à un débat national sur des choix fondamentaux de société. Ces choix portent soit sur un modèle d'agriculture spécialisée et productiviste sur les meilleures terres au risque d'exclure les moins compétitifs, soit sur un modèle de développement local basé sur des formes de production agricole favorisant intensification écologique (Griffon, 2006) et cohésion sociale. Un choix entre ségrégation et intégration... (Hubert, Caron, 2009).

Il est encore temps, sinon vital, d'engager une réflexion sur les choix de développement à Madagascar, en transparence et en fonction de données révisées et fiables. Les projets de Daewoo et de Varun ont vraisemblablement échoué mais d'autres investisseurs, venant de pays émergents ou industrialisés, motivés par de simples spéculations ou par des stratégies d'anticipation de crises alimentaires et énergétiques, tentent de contrôler à leur tour des espaces et des ressources naturelles ${ }^{69}$. La question des grandes appropriations foncières se posera de nouveau. La mise au point de la nouvelle politique foncière se doit d'intégrer cette nouvelle donne et de prévoir un cadre d'accueil des investissements fonciers, conforme à une déontologie internationale en cours de conception (de Schutter, 2009) et de concert avec les populations titulaires de droits sur les espaces convoités ${ }^{70}$. Cet exercice de concertation territoriale n'est pas à considérer comme une simple formalité dont il faut s'acquitter; il est un gage d'efficacité et de durabilité des politiques publiques. Le seul effet positif des expériences malheureuses de Daewoo et de Varun est d'avoir démontré que le traitement dans l'opacité de projets de cette importance peut nuire non seulement aux populations concernées, mais également à l'investisseur et au pouvoir en place.

\section{BIBLIOGRAPHIE}

Amelot, X., Moreau, S., Carrière, S. 2008. Des justiciers de la biodiversité aux injustices spatiales. L'exemple de l'extension du réseau d'aires protégées à Madagascar. Colloque International «Justice et Injustice spatiales ", 12-15 mars 2008, Université Paris X Nanterre.

Bertrand A., Karpe P., Aubert S., 2008. Le statut juridique des terres : perspectives historiques et sociales. In Sandron F., Population rurale et enjeux fonciers à Madagascar. CITE / Karthala, Antananarivo, p. 35-57.

EchoGéo, 11 | 2010 
Blanc-Pamard Ch., 1986. Dialoguer avec le paysage ou comment l'espace écologique est vu et pratiqué par les communautés rurales des hautes terres malgaches. Milieux et paysages, Masson, p. 17-35.

Cotula L., Vermeulen S., Leonard R., Keeley J., 2009. Land grab or development opportunity? Agricultural investment and international land deals in Africa. IIED, FAO, IFAD, $111 \mathrm{p}$.

D’Ersu L., 2009. Changement d'homme providentiel à Madagascar. Etudes, novembre 2009, p. 451-461.

De Schutter, 2009. Contrats d'acquisition ou de location à large échelle de terres agricoles : principes et mesures pour relever le défi des droits de l'homme. Nations Unies, 11 juin 2009

ECR, 2006-2008. Les données de référence sur les coûts et temps moyens requis pour l'exécution des procédures foncières. Millennium Challenge Account, Antananarivo. 45 p.

Grain, 2008. Main basse sur les terres agricoles en pleine crise alimentaire et financière. 15 p. http/ www.grain.org/go/mainbasse

Griffon M., 2006. Nourrir la planète. Pour une révolution doublement verte. Odile Jacob, 456 p.

Haralambous S., Liversage H., Romano M., 2009. The growing demand for land. Risks and opportunities for smallholders farmers. IFAD, 32d session of Governing Council, Rome, $17 \mathrm{p}$.

Hubert B., Caron P., 2009. Imaginer l'avenir pour agir aujourd'hui, en alliant prospective et recherche : l'exemple de la prospective Agrimonde. Nature, Sciences, Sociétés. En cours de publication

Ministère de l'Agriculture, de l'Elevage et de la Pêche, 2005. Lettre de Politique Foncière. Programme National Foncier, Antananarivo, $12 \mathrm{p}$.

Marchal J.-Y., 1974. La colonisation agricole au Moyen-Ouest malgache. La petite région d'Ambohimanambola. Atlas des structures agraires à Madagascar. Orstom, Maison des Sciences de l'Homme. Mouton, 122 p. + cartes.

Moreau S., 2009. Mutations des relations à la terre et devenir des paysanneries dans le contexte d'ouverture de Madagascar à la mondialisation. Echogéo.

Pellerin M., 2009. Madagascar : un conflit d'entrepreneurs ? Figures de la réussite économique et rivalités politiques. Politique Africaine, $n^{\circ} 113$, mars 2009.

Raison J.-P., 1969. Les travaux du service topographique de Madagascar et leur utilisation en sciences huamines. Madagascar, revue de Géographie, n¹4, p. 97-129.

Raison J.-P., 1984. Les Hautes Terres de Madagascar. Orstom / Karthala, 2 tomes, 662 p. et 614 p. Raison J.-P., 1991. Dynamismes ruraux et contrastes fonciers dans Madagascar en crise. Revue Tiers-Monde, t. XXXII, n¹28, p. 901-915.

Rakoto Ramiarantsoa H., 1991. La dynamique des paysages sur les Hautes Terres centrales malgaches et leur bordure orientale. Thèse nouveau régime de géographie, Université de Paris X, 321 p. + annexes.

Rakoto Ramiarantsoa H., 2008. Madagascar au XXIème siècle. La politique de sa géographie. Echogeo, ${ }^{\circ} 7$, http://echogeo.revues.org/index8753.html

Rochegude A., 2008. La réforme foncière à Madagascar. Relire le droit de propriété sur la terre. In Barrière O., Rochegude A., Foncier et environnement en Afrique. Des acteurs aux droits. Cahiers d'Anthropologie du droit, Karthala, Paris, p. 215-248. 
Romer P., 2008. Using gateways cities to accelerate economic development. Stanford University, 7 p. Teyssier A., Raharison H., Ravelomanantsoa Z., 2007. La réforme foncière malagasy ou le pari de la compétence locale. FAO, Land Reform Bulletin, Rome, 2007-1, p. 34-49.

Teyssier A., Ravelomanantsoa Z., 2008. Madagascar. Une réforme face à la faillite du système Torrens. Etudes Foncières, n¹34, p. 34-38.

Teyssier A., Andrianirina Ratsialonana R., Razafindralambo R., Razafindrakoto Y., 2009. Décentralisation de la gestion des terres à Madagascar : processus, enjeux et perspectives d'une nouvelle politique foncière. IRD / Karthala, p. 273-297.

Uellenberg A., 2009. Country Study « Foreign Direct Investment in Land" - Madagascar. PGME / GTZ, Antananarivo, juin 2009. $80 \mathrm{p}$.

\section{NOTES}

1. Révélé par l'ONG Grain dès octobre 2008 (Grain, 2008). Plusieurs études ont ensuite commencé à traiter ce sujet (Cotula et al., 2009, Haralambous et al., 2009,...)

2. Appropriation, accaparement, acquisition, contrôle, location,... les mots font débat. Dans le cas de Daewoo et de Varun, la prise de contrôle de terres ne correspond pas à proprement parler à une obtention de droits de propriété. Néanmoins, si l'on rapporte la durée des contrats et des baux envisagés (50 à 99 ans) à l'espérance de vie moyenne d'un cultivateur malgache (59 ans selon l'UNICEF), force est de constater qu'un adulte sera définitivement dépossédé de son terrain. Aussi, nous utiliserons le terme "appropriation» pour désigner ces projets de transactions foncières.

3. Javier Blas. Land leased to secure crops for South Korea. Financial Times, London, 18/11/08.

4. Une liste impressionnante d'infrastructures à la charge de Daewoo a été communiquée : 1.170 écoles, 170 cliniques, 250 marchés, 120 églises, 60 stations électriques, 8 aéroports, 30 usines et silos, 8 ports,...

5. Citons entre autre : Jean-Pierre Tuquoi. La Corée du Sud relance la course aux terres agricoles. Le Monde, 20/11/08 - Graham Bowley. Short of Food? Rent Half a Country. New York Times, 20/11/08.

6. Sylvain Amiotte. Daewoo s'apprête à coloniser Madagascar. Clicanoo, 15/12/08 - Daewoo fait main basse sur la terre. Collectif de Défense des Terres Malgaches - Doan Bui. Daewoo se paie Madagascar. Le Nouvel Observateur, 24/12/2008.

7. Javier Blas. Welcome fades for wealthy nations. Financial Times, 20/11/08. Laetitia Clavreul. Des opportunités et des risques. Le Monde, 20/11/08.

8. Le Monde du 29/1/09 présente une carte des régions où des terres sont "déjà louées" par Daewoo).

9. Dont l'influent Collectif de Défense des Terres Malgaches, créé en décembre 2008 en réaction au projet Daewoo et qui invite à signer une pétition contre ce projet dès le 05/1/09 http:// terresmalgaches.info/ Il faut également signaler les Amis de la Terre, Action Aid, la Confédération Paysanne, Peuples Solidaires,... Ces organisations ont adressé des courriers de protestation auprès du directeur général de Daewoo Logistics et du gouvernement malagasy.

10. "L'affirmation sur l'attribution à titre gratuit d'un terrain d'une superficie de 1,3 millions d'hectares à la société Daewoo Logistics est totalement fausse ».

11. Terrains pour Daewoo. Et voici la preuve de son existence. La Gazette de la Grande Ile, 25/11/08. Informations également parues dans La Vérité du 16/12/08.

12. Une forte délégation de fonctionnaires sud-coréens, en partie supportée par Daewoo, s'est rendue à Madagascar en décembre 2008 pour présenter un ensemble d'actions de coopération 
(Ministry of Land, Transport and Maritime Affairs. Korea-Madagascar Construction Cooperation Forum, Antananarivo, 15/12/08).

13. Les investisseurs étrangers n'ont pas le monopole de ce discours. Des sites internet malgaches militent pour une plus grande ouverture aux investissements étrangers et souhaitent voir levées toutes les contraintes d'accès de la terre aux étrangers. Malagasy land and foreign investments, 21/10/08 http://andrydago.wordpress.com/2008/10/21/malagasy-land-andforeign-investments/

14. http://faostat.fao.org/default.aspx

15. Les Nouvelles, $18 / 11 / 08$.

16. Marchal, 1974 ; Raison JP, 1984 ; Blanc-Pamard, 1986 ; Rakoto-Ramiarantsoa, 1991,...

17. Selon le quotidien Joongang Ilbo, "il s'agit la plupart du temps de terres non défrichées. De plus, des emplois vont être créés et $30 \%$ des bénéfices iront au gouvernement local. [...] Les Anglais disposent à Madagascar d'une immense ferme produisant de l'huile de jatropha destinée à la fabrication de biocarburant. Sont-ils bien placés pour accuser de "néocolonialisme" la Corée du Sud, pays importateur de céréales, qui veut simplement garantir la subsistance de sa population ?». Daewoo Logistics "envisage d'investir à Madagascar quelque 6 milliards de dollars sur vingt ans, ce qui est énorme, comparé à l'aide du FMI dont bénéficie le pays, soit 0,9 milliard de dollars ». "Cela vient-il de la cupidité des Européens, qui sont en train de perdre leur influence sur le continent africain ?» s'interroge le quotidien Maeil Kyongje. Jeong Eun-Jin. Corée du Sud - Néo-colonialisme ou aide au développement ? Courrier International, 20/11/08.

18. Daewoo : To be or not to be a scandal? www.madagascar-tribune.com, 23/6/09. NasoloValiavo Andriamihaja. La souveraineté nationale ne nourrit personne. L'Express, 29/10/09.

19. bekotopaysan.blogspot.com

20. Citons entre autres www.sobika.com

21. Communiqué du 01/12/08 signé par FIFATA, Koloharena, Réseau SOA, la Chambre d'Agriculture Nationale, la Coalition Paysanne de Madagascar et la Confédération Nationale Sahavanona.

22. Sefafi (Sehatra Fanaraha-Maso ny Fiainam-Pirenena - Observatoire de la Vie Publique). Comment gérer nos terres ? 08/12/08.

23. Programme Alimentaire Mondial des Nations Unies - http://www.wfp.org/countries/ madagascar

24. L'atteinte à la terre des ancêtres renvoie à un rapport ancestral à la terre qui tend à exclure l'étranger au sens large, comme le rappelle JP Raison : « une règle fondamentale : la terre ne peut être cédée aux étrangers, non seulement aux Européens, mais aux Malgaches originaires d'autres régions " (Raison 1991). La notion d'un tanindrazana inaliénable (Bertrand et al., 2008) témoigne d'un traumatisme historique mais elle a également pu être galvaudée à dessein : «La hantise de la cession de la terre aux étrangers est apparue avec beaucoup de force, montrant combien le traumatisme de la colonisation est encore vif. [...]D'autres insistent sur la xénophobie masquée derrière l'attachement à la représentation traditionnelle de la terre, et l'instrumentalisation politique de l'affaire Daewoo dans une stratégie de conquête du pouvoir ». (Moreau, 2009)

25. Rémi Carayol. La grande braderie de l'île. Libération, 24/2/09.

26. Cette formule est extraite d'un discours de Andry Rajoelina le 26/1/09, où il a été fait allusion à l'assassinat de Radama II en 1863, suite à la Charte Lambert de 1855 qui rendait la terre accessible aux étrangers (Pellerin, 2009).

27. Et cela, moins de trois semaines après l'annonce de l'accord à Séoul... Christian Oliver. Daewoo unsure of Madagascar deal. Financial Times, 05/12/08.

28. Jullian Ryal, Mike Pflanz. Land rental deal collapses after backlash against "colonialism". Telegraph, 14/1/09.

29. Mobilisation réussie pour Andry Rajoelina à Ambohijatovo. www.tribune-madagascar.com. $18 / 1 / 09$. 
30. Daewoo Logistics goes to bankrupt. Fairplay, 06/7/09.

31. Olivier Ramaro, Antony Sguazzin. Madagascar may welcome farms ventures after Daewoo cancellation. 10/4/09. www.bloomberg.com/apps/news?pid=20601116\&sid=acmiQQBrFzbI Cet article va inciter les organisations militantes à maintenir leur vigilance (voir notamment la « protestaction » sur www.regenwald.org: Daewoo's rainforest land grab in nature's paradise, 19/6/09)

32. Sébastien Hervieu. A Madagascar, une société indienne compte louer près de 500000 hectares. Le Monde, 20/3/09. L'Inde guigne à son tour des terres arables à Madagascar. Le Monde, $21 / 3 / 09$.

33. Une autre filiale de Varun, Varun Energy Corp., a obtenu des permis miniers pour l'exploitation d'uranium à Mangataboahangy (région de l'Amoron'i Mania) et Ankilizato (région du Menabe) dans le cadre d'une convention avec deux organismes gouvernementaux malgaches : l'Office des mines nationales et des industries stratégiques (Omnis) et l'Institute for the Science and Technique of Nuclear Energy (INSTN), dépendant du Ministère de l'enseignement supérieur et soutenu par l'Agence internationale de l'énergie atomique (AIEA) (Varun International. Lettre de l'Océan Indien, $\left.{ }^{\circ} 1268,05 / 9 / 09\right)$. Varun Petroleum Madagascar, enregistrée à l'Ile Maurice, a également obtenu un bloc de prospection pétrolière onshore à Madagascar de plus de $5.000 \mathrm{~km} 2$ au sud de Mahajanga (Madagascar : un bloc pétrolier pour Varun International. Lettre de l'Océan Indien, $\left.\mathrm{n}^{\circ} 1267,31 / 8 / 09\right)$.

34. Source : termes de référence entre Varun et Sodhai.

35. Ce qui équivaut à la moitié de la production annuelle nationale de paddy !

36. Lentille consommée en Inde.

37. Source : Contract farming between Varun Agriculture Sarl and each association of 13 different plains. Antoshihy, 26/1/09, 8 p.

38. Aucun document contractuel n'apporte de précision sur ce point.

39. Le Collectif de Défense des Terres Malgaches a publié une newsletter $n^{\circ} 7$ demandant une clarification sur les suites données au projet Varun. Ce bulletin a été repris par différents organes de presse nationaux et internationaux (Pour la défense des paysans et des terres malgaches : le contrat d'agri-business de Varun doit être annulé. Le Courrier de Madagascar, 12/10/08. Les méthodes de l'indien Varun à Madagascar. Courrier International, 28/10/09).

40. Lantoniaina Razafindramiadana. Les terres inaccessibles aux étrangers. L'Express, 19/8/09. Notons que cet article prend le contrepied d'un article du même auteur paru un mois plus tôt : Lantoniaina Razafindramiadana. La terre accessible aux étrangers. L'Express, 03/7/09.

41. Ce qui n'est pas garanti, puisque les plans de repérage relatifs aux demandes d'acquisition de terrains domaniaux montrent des hameaux inclus dans les zones pré-délimitées.

42. "C'est un projet gagnant-gagnant. Ils se sont engagés à électrifier les campagnes, acheminer l'eau potable, et construire des écoles et des centres de santé." André Randriamanesy, chef de région de la Sofia, cité par S. Hervieu en mars 2009.

43. "Comment peut-on pousser à des décisions sur un projet d'une si grande ampleur alors que tous les paysans ne sont pas au courant et que l'on n'a pas accès au détail des engagements ?" Léon Mananjara, Directeur du Développement Régional de la Sofia cité par S. Hervieu en mars 2009.

44. Chido Makunike. The lessons of Daewoo's failed Madagascar land lease deal. African Agriculture, 15/2/09.

45. «On ne sait rien de ce projet et on nous demande de le faire accepter par la population. [...] On m'a demandé d'aller voir les paysans pour leur faire accepter l'opération. Mais si j'y vais, je me fais lyncher. » Philibert Randriamaharitra, chef de district de Toamasina II. Cité par Rémi Carayol. La grande braderie de l'île. Libération, 24/2/09. Citation également par Michel Beuret. Le dragon chassé de la Grande Ile. L'Hebdo, 03/9/09. 
46. Jean-Claude Ramandimbiarison, in Hélèna Razafiarisoa. Madagascar: Ravalomanana à l'heure du bilan. Syfia Madagascar, 27/10/06.

47. Lors du Congrès Mondial sur les Parcs à Durban, le président Ravalomanana a fait part le 17 septembre 2003 de sa décision d'augmenter la surface des aires protégées de 1.700 .000 ha à 6.000.000 ha en cinq ans. Un groupe Vision Durban a été chargé de la délimitation de nouvelles aires protégées mais la concertation avec les populations résidentes a été négligée dans bien des cas (Amelot et al., 2009).

48. Des Schémas Régionaux d'Aménagement du Territoire sont en cours dans plusieurs régions. Selon les démarches employées, leur conception accorde une importance variable aux représentations et aux projets des acteurs locaux.

49. L'EDBM est une Agence de Promotion des Investissements, organisme autonome agissant au nom de l'Etat malgache et rattaché à la Présidence de la République. Il a été soutenu en partie par la Banque Mondiale.

50. Loi n²007-036 du 14 janvier 2008 sur les investissements à Madagascar.

51. La loi $n^{\circ} 2003-028$ a ouvert un premier accès des étrangers à la propriété foncière en autorisant l'acquisition «de biens immobiliers sur présentation d'un programme d'investissement». L'accès à la propriété était conditionnée au dépôt d'un projet d'investissement d'au moins 500.000 \$ et à sa validation par une autorité aux contours mal définis. Très peu d'investisseurs ont acquis des terrains selon cette procédure, mais cette ouverture frileuse à la propriété a néanmoins provoqué des réactions hostiles du parlement et de l'opinion.

52. Il suffit de remplir une demande sur un formulaire de l'EDBM, de présenter l'activité prévue, de fournir un certificat de situation juridique si l'immeuble est immatriculé, un plan et d'autres pièces exigées habituellement par les Domaines. Cette différence de traitement entre les investisseurs et les usagers tenus de suivre les 24 étapes des procédures d'immatriculation a provoqué des frustrations, exprimées lors des manifestations de 2009.

53. Des projets de coopération sud-coréens et indiens ont commencé à concevoir fin 2008 des appuis aux services fonciers, dans l'espoir d'une réactivation de leur fonctionnement.

54. Lettre de Politique Foncière du 03/5/05 (Maep, 2005).

55. Loi n²005-019 du 17 octobre 2005, loi n²006-031 du 24 novembre 2006 fixant le régime juridique de la propriété foncière privée non titrée, et son décret d'application n²007-1109 du 18 décembre 2007.

56. Comme le titre foncier est maintenu, deux systèmes de validation des droits sur la terre se combinent: la formalisation de la propriété titrée par des titres fonciers relève de la compétence de l'administration foncière, la formalisation de la propriété non titrée par des certificats fonciers relève d'une nouvelle compétence des communes.

57. Notable, ancien, connu pour son expérience et sa sagesse présumée.

58. 12 bailleurs de fonds ont soutenu la nouvelle politique foncière malgache et lui ont alloué près de 45.000.000\$ de 2005 à 2009 .

59. Ainsi, près d'un tiers des 1.550 communes malgaches dispose d'un guichet foncier.

60. Loi sur le Domaine public de l'Etat $n^{\circ} 2008-13$ du 23 juillet 2008 et son décret d'application

61. Loi sur le Domaine privé de l'Etat, des Collectivités Décentralisées et des personnes morales de droit public, $n^{\circ} 2008-14$ du 23 juillet 2008.

62. Les travaux du Stanford Center for International Development ont été diffusés par des conseillers de la Présidence.

63. Traduction de gateway cities ou charter cities. http://www.chartercities.org/concept

64. "The developing nation that wants to serve as host to a new gateway city would deed an uninhabited piece of land to the development corporation. The host would retain sovereignty, but exempt it from all its usual laws and registration. Ideally, this would be on a coast and permit the construction of a port. It would be large enough to support the construction of a city with 
millions of residents at reasonable densities perhaps between 700 and 1000 square kilometers." (Romer, 2008)

65. Ministry of Land, Transport and Maritime Affairs. Korea-Madagascar Construction Cooperation Forum, Antananarivo, 15/12/08

66. Plan d'Action pour Madagascar, 2007-2012. 114 p. www.madagascar.gov.mg/MAP

67. Slogan du Millennium Challenge Account (MCA), principal partenaire financier de la réforme foncière malgache, dont les activités ont été suspendues en juillet 2009 suite aux sanctions américaines vis-à-vis du gouvernement de transition.

68. Les investissements fonciers à grande échelle. Quelles réalités? Quelles conséquences pour l'agriculture familiale et quels choix de développement? Conférence du Cirad, Montpellier, 03/9/09.

69. L'eau est également recherchée, notamment par l'Arabie Saoudite qui a eu pour projet de pomper et d'exporter l'eau de deux fleuves de la Côte Est. Fanja Saholiarisoa. Vente d'eau. Des opérateurs arabes en prospection. L'Express, 07/7/09. Ce projet a immédiatement été dénoncé par le parti écologiste malgache, Hasin'i Madagasikara.

70. Olivier Rabaey. Vendre ou donner des terres en location. Oui, mais pas n'importe comment. Tribune, 12/11/09.

\section{RÉSUMÉS}

Les manœuvres d'appropriation foncière à grande échelle tentées fin 2008 par les entreprises Daewoo Logistics et Varun International, largement relayées par les médias et dénoncées par diverses organisations, ont participé à la déstabilisation du gouvernement Ravalomanana. Les montages de ces deux grands projets agro-industriels ont suivi des trajectoires différentes, l'un choisissant de contrôler d'immenses superficies par bail emphytéotique, l'autre privilégiant des formes de contractualisation de la production, mais tous deux ont été abandonnés face à des mouvements de contestation basés sur l'inaliénabilité de la «terre des ancêtres ». Le rejet de ces projets d'envergure encore inédite et en partie tournés vers des cultures d'exportation est compréhensible tant les retombées économiques et sociales paraissaient incertaines. Madagascar ne saurait néanmoins se priver d'investissements dans le secteur agricole, mais des choix de développement, orientés par une meilleure connaissance des processus en cours, restent à opérer en toute transparence, afin de combiner développement de l'agro-business et promotion des exploitations familiales. Pour l'instant, ces incertitudes marquent une politique foncière qui, même rénovée, hésite entre la formalisation de droits sur le sol au profit du plus grand nombre grâce à une décentralisation de la gestion foncière et l'octroi de vastes espaces à des firmes internationales suivant des procédures accélérées.

\section{INDEX}

Mots-clés : appropriation foncière, Daewoo, développement rural, Madagascar, politique foncière, Varun 


\section{AUTEURS}

\section{ANDRÉ TEYSSIER}

André Teyssier est membre du Cirad-UMR Tetis, Observatoire du Foncier ateyssier@cirad.fr

\section{LANDRY RAMAROJOHN}

Landry Ramarojohn est membre du Programme National Foncier landry141270@yahoo.fr

\section{RIVO ANDRIANIRINA RATSIALONANA}

Rivo Andrianirina Ratsialonana est membre de l'observatoire du Foncier ratsialonana@yahoo.fr 